\title{
Ação educativa com idosos institucionalizados
}

\author{
Gabriela Nobile Bublitz ${ }^{1}$, Jéssica Andrade de Alencar $^{1}$ e Denise Andreo ${ }^{2}$
}

A deficiência nutricional em idosos vem aumentando juntamente com os transtornos alimentares e pode levar o indivíduo a diversos quadros, como: desnutrição, obesidade, hipertensão, diabetes, doenças cardiovasculares e a perda de apetite, que tem sido relacionada à ausência de elementos dentários, e que leva à diminuição do consumo de carnes e hortifrútis. Considerando ainda as demências, notadamente a Doença de Alzheimer, este quadro tornase ainda mais agravante. Este estudo objetivou incentivar o consumo de hortifrútis em idosos institucionalizados, para que estes possam ter uma dieta balanceada, de acordo com as necessidades nutricionais nesta fase do ciclo da vida. O presente trabalho foi realizado em uma Instituição de Longa Permanência (ILP) para idosos, no interior de São Paulo, pela equipe do Programa Mesa Brasil Sesc Rio Preto. Para incentivar a mudança no hábito alimentar, optou-se por dinâmicas que favoreçam a coordenação motora e a memorização, com o objetivo de evidenciar a importância dos hortifrútis para a saúde. O objetivo foi alcançado, pois o grupamento entendeu a importância de ingerir hortifrútis e como montar um prato saudável e completo nutricionalmente.

Palavras-chave: alimentação, Instituição de Longa Permanência para Idosos, idosos, programa social, transtornos alimentares.

\section{Educational activities with institutionalized elderly}

The nutritional deficiency among the elderly is increasing along with the eating disorders, which can lead to many health problems, such as: malnutrition, obesity, hypertension, diabetes, cardiovascular diseases and loss of appetite, which is related to the lack of dental elements that causes a decrease in the consumption of meat, fruits, and vegetables. Also, considering dementia, being Alzheimer the most common to be analyzed in the research institute, the situation becomes even more aggravating. This research aimed at the incentive for the consumption of fruits and vegetables by the institutionalized elderly, so that they can have a complete and balanced diet according to their nutritional necessities. The current study was performed in a nursing home in São Paulo by the program Mesa Brasil Sesc Rio Preto. To encourage the change in the eating habits, dynamics that help coordination and memorization were used to highlight the importance of fruits and vegetables in human health. The goal was reached, once they understood the importance of the healthy eating habits and how to include a significant amount of nutrients in the diet.

Keywords: eating, Nursing Homes, elderly, social program, eating disorder.

\footnotetext{
${ }^{1}$ Alunas do Curso de Graduação em Nutrição, na Universidade Paulista, Campus JK.
}

${ }^{2}$ Nutricionista, Mestre em Engenharia e Ciência de Alimentos e Coordenadora do Programa Mesa Brasil Sesc Rio Preto. Endereço para correspondência: Av. Francisco C. Oliveira, 1.333, Chácara Municipal, São José do Rio Preto - SP. Telefone para contato: (17) 3216-9366 - Fax: (17) 3227-6104. E-mail: denise@riopreto.sescsp.org.br 


\section{INTRODUÇÃO}

Entende-se por envelhecimento as alterações fisiológicas que acontecem ao longo do tempo em organismos multicelulares. Tais alterações acontecem nas moléculas e nas células que acabam por prejudicar o funcionamento dos órgãos e do organismo em geral. Podem-se dividir as causas em genética, estilo de vida e o meio ambiente em que a pessoa vive ${ }^{[1]}$.

O envelhecimento afeta diretamente o estado nutricional do indivíduo devido a todas as alterações que ocorrem no organismo, tais como: diminuição dos botões gustativos, redução do olfato e paladar, diminuição da secreção salivar e gástrica, falha na mastigação (pela ausência de dentes e/ou próteses mal adaptadas), constipação intestinal devido à redução da motilidade. Estudos epidemiológicos também indicam que, especialmente em homens idosos, a desnutrição reduz significativamente o tempo de vida [2].

Do mesmo modo, a educação nutricional permite que o idoso se mantenha distante do risco de desnutrição ou mesmo de doenças advindas por excessos alimentares, como por exemplo, a obesidade, a hipertensão, o diabetes e doenças cardiovasculares [3].

A educação nutricional é definida como um processo ativo que envolve mudanças no modo de pensar, sentir e agir dos indivíduos e pelo qual eles adquirem, mudam ou reforçam conhecimentos, atitudes e práticas conducentes à saúde. Trata-se de ferramenta que dá autonomia, para que a pessoa possa assumir, com plena consciência, a responsabilidade por seus atos relacionados à alimentação [4].

Os aspectos relativos à alimentação oferecida pelos abrigos geriátricos são essenciais, considerando o grande impacto dos hábitos alimentares do idoso no seu estado de saúde. A Portaria no 810, do Ministério da Saúde, sancionada em 1989, estabelece as normas para o funcionamento de instituições geriátricas e destaca a responsabilidade da própria instituição no que diz respeito à produção de refeições para os internos [5]. Dessa forma, o planejamento adequado da alimentação, considerando as características nutricionais e higiênicosanitárias, cabe aos responsáveis pelo setor de produção de refeições do local [6].

A mastigação é importante para uma boa nutrição no idoso. Com o envelhecimento, os hábitos de mastigação mudam, acentuadamente, tanto nos homens como nas mulheres. Essas alterações na capacidade mastigatória do idoso são devidas ao aparecimento frequente de cáries e doenças periodontais; às próteses totais ou parciais inadaptadas ou em péssimo estado de conservação, e à ausência de dentes. Esses fatores interferem no comportamento inicial do processo digestivo, favorecendo sua inadequação tanto no aspecto enzimático como no mecânico [7].

Sabe-se que o edentulismo não é consequência natural do envelhecimento, e que os dentes naturais, quando bem tratados, podem permanecer em funcionamento por toda a vida. As principais causas de ausência de dentes e de uso de próteses totais na terceira idade são decorrentes de cáries não tratadas e da periodontite, embora essas causas possam ser preveníveis com a adoção da tecnologia disponível[ ${ }^{[8]}$.

A perda de apetite em idosos tem sido, geralmente, relacionada com ausência de elementos dentários e com o uso de próteses [9. As pessoas que usam dentaduras mastigam 75 a $85 \%$ menos eficientemente que aquelas com dentes naturais, o que leva à diminuição do consumo de carnes, frutas e vegetais frescos, razão porque idosos com próteses totais tendem a consumir alimentos macios, facilmente mastigáveis, pobres em fibras, vitaminas e minerais, fato que pode ocasionar consumo inadequado de energia, ferro e vitaminas ${ }^{[10]}$.

Os idosos apresentam condições peculiares que condicionam o seu estado nutricional. Alguns desses condicionantes são devidos às alterações fisiológicas próprias do envelhecimento, enquanto outros são influenciados pelas enfermidades presentes e por fatores relacionados com a situação socioeconômica e familiar [7].

Com o envelhecimento, a nutrição torna-se especialmente importante, em função das modificações sensoriais, gastrintestinais, metabólicas, neurológicas, desenvolvimento de doenças crônico-degenerativas, que interferem diretamente no consumo de alimentos [1].

Uma consequência do processo de envelhecimento populacional é o aumento significativo na prevalência de doenças crônico-degenerativas. Dentre elas, destacam-se as demências, sendo a mais 
comum a Doença de Alzheimer (DA). Alguns estudos epidemiológicos indicam que a prevalência da DA dobra a cada cinco anos em pessoas com idades entre 65 e 85 anos. A DA caracteriza-se por um declínio insidioso, progressivo da memória e de outras funções corticais, como linguagem, conceito e julgamento [11].

$\mathrm{Na}$ Doença de Alzheimer também têm sido observados muitos transtornos alimentares, tanto no sentido da anorexia, como no da hiperfagia. Uma das alterações mais típicas talvez seja a alteração da preferência alimentar, como por exemplo, a súbita preferência pelos doces, antes indiferentes. Não estão claras quais são as causas dessas mudanças, mas elas podem se relacionar com mudanças biológicas internas e com mudanças ambientais. Os pacientes têm menor capacidade de detectar as sensações internas e externas da necessidade de comida ou da saciedade da fome ${ }^{[12]}$.

O Mesa Brasil é uma iniciativa do Serviço Social do Comércio (Sesc), cujo objetivo é o combate à fome e ao desperdício de alimentos. Este Programa existe no Brasil todo desde 2003 e, no Estado de São Paulo, desde 1994 [13]. Está presente em 13 unidades do Sesc São Paulo, sendo: São José do Rio Preto, Bauru, Ribeirão Preto, Piracicaba, Campinas, Taubaté, Santos, Osasco, São José dos Campos, Santo André e na grande São Paulo: Interlagos, Carmo e Itaquera.

Em São José do Rio Preto, o Mesa Brasil atende 52 instituições sociais, que estão cadastradas para receber pelo menos uma vez por semana, doações de alimentos que complementam as refeições dos assistidos. Em média, são cerca de 10.000 pessoas atendidas mensalmente.

Com o objetivo de promover o conhecimento e a mudança de hábitos alimentares, tem sido realizada uma série de ações educativas, direcionadas aos manipuladores de alimentos e demais profissionais, como: oficinas de Boas Práticas de Fabricação, oficinas culinárias utilizando receitas com aproveitamento integral de alimentos, elaboração de cardápios, organização de estoque, congelamento de alimentos, além de atividades de educação nutricional com o público atendido nas instituições sociais [13].

O Programa integra doadores parceiros, entidades sociais do município e voluntários, com vistas ao combate à fome e ao desperdício, arrecadando alimentos onde "sobra" e entregando onde "falta", por meio do sistema de colheita urbana [13].
Considerando todos os aspectos citados anteriormente, observou-se a necessidade da realização do presente trabalho, envolvendo idosos institucionalizados em uma casa de repouso, atendida pelo Programa Mesa Brasil Sesc Rio Preto.

\section{OBJETIVOS}

Os principais objetivos deste trabalho foram:

- estimular o consumo de hortifrútis diversificados, visando uma dieta completa e balanceada, de acordo com as necessidades nutricionais nesta fase do ciclo da vida;

- incentivar a realização de atividades educativas e lúdicas periodicamente, com os idosos atendidos nas Instituições Sociais, proporcionando condições para que possam associar o aprendizado, a escolhas alimentares mais saudáveis em sua rotina diária.

\section{METODOLOGIA}

O presente trabalho foi realizado pela equipe do Programa Mesa Brasil Sesc em parceria com uma Instituição de Longa Permanência de Idosos, no município de São José do Rio Preto - SP, nos meses de abril e maio de 2014. Dentre os 41 idosos, 13 foram selecionados para participar (de ambos os sexos), escolhidos pela nutricionista do local, conforme a situação mental e física. Foram excluídos os idosos que não tinham condições motoras e psicológicas para participarem.

Antecedendo o início das atividades, foi realizada a análise do almoço e café da manhã, consumidos pelos idosos.

$\mathrm{Na}$ sequência, foram coletadas informações dos prontuários dos 13 idosos selecionados, como nome, idade e estado nutricional. Durante a coleta, foi possível observar que nenhum possui filhos e todos são solteiros. Além disso, verificou-se que a maioria possui a doença de Alzheimer, impossibilitando-os de morarem sozinhos. Supõem-se então, que estes são os motivos pelos quais permaneciam institucionalizados.

Mediante as observações realizadas durante as refeições dos idosos concluiu-se que aqueles que estão com sobrepeso não respeitam a dieta, manifestando o interesse em repetir as preparações, excedendo a 
ingestão calórica adequada. Já os que estão com baixo peso, costumam ingerir quantidade insuficiente de alimentos, prejudicando assim a ingestão adequada de nutrientes.

Foi possível notar ainda, que dentre a população estudada, alguns rejeitam hortifrútis, não provando, dizendo não gostar, devido à cor forte, como a beterraba, por exemplo.

Para incentivar a mudança no hábito alimentar, optou-se por dinâmicas que favorecessem a coordenação motora e a memorização. Não foram utilizados outros métodos como palestras, devido à importância de utilizar frases curtas e objetivas para garantir a atenção e não confundí-los.

Foram realizadas duas atividades com o objetivo de explicar a importância da ingestão de hortifrútis e criar condições para que escolhas saudáveis sejam realizadas na rotina diária das refeições dos idosos, já que somente a presença do nutricionista para a elaboração de cardápios completos nutricionalmente não é suficiente para a mudança de hábitos alimentares.

Ao final do trabalho, a equipe do Programa Mesa Brasil deixou as orientações e o material no local, para que houvesse continuidade das atividades, como forma de estimular esse tipo de ação educativa com os idosos.

\section{Dinâmicas}

\section{Bingo dos Hortifrútis}

Objetivo: transmitir informações sobre a importância nutricional dos hortifrútis à saúde do idoso.

Conteúdo Programático do Encontro: os idosos encontravam e marcavam a figura do hortifrúti nas cartelas, com a ajuda dos voluntários e profissionais que estavam presentes, conforme um integrante da equipe do Programa Mesa Brasil citava o nome do hortifrúti sorteado dentro de uma caixa. Enquanto as figuras eram buscadas nas cartelas, houve uma rápida explicação sobre a importância e os benefícios de sua ingestão.

Estratégias e recursos: os números das cartelas foram substituídos por imagens de frutas e legumes. Foi entregue a cada idoso uma cartela, para que eles colocassem um grão de feijão na figura de cada alimento sorteado. As figuras de alimentos foram sorteadas dentro de uma caixa e, durante a marcação, explicavamse os benefícios e seus principais nutrientes. Para garantir o consumo de frutas, todos foram vencedores do bingo. Conforme as cartelas foram completadas, cada participante escolhia como prêmio, a fruta de sua preferência.

Recursos Humanos: nutricionista e estagiárias do Programa Mesa Brasil e equipe de profissionais do local.

Recursos Materiais: Cartelas de bingo com figuras aleatórias de hortifrútis, grãos de feijão e frutas: tangerina (ponkan), goiaba, maçã, mamão, caqui, manga, laranja e banana.

\section{Prato Saudável}

Objetivo: ensinar os idosos quanto a montagem de um prato saudável, com uma refeição completa e nutritiva.

Conteúdo Programático do Encontro: foi explicado sobre a importância de realizar uma refeição completa, contendo todos os grupos do guia alimentar nutricional.

Estratégias e recursos: foram utilizadas figuras de alimentos saudáveis e não saudáveis. Todas foram colocadas aleatoriamente no centro da mesa. Os idosos sentaram-se ao redor e recebiam um prato de acrílico, pincel e cola. Eles deveriam escolher os alimentos e, na sequência, colar no prato para montar uma refeição saudável e completa nutricionalmente. Para isso, cada participante recebia o auxílio dos profissionais na seleção e colagem das figuras. Durante a atividade, o nutricionista fazia explicações sobre os alimentos e nutrientes, orientando-os na escolha de alimentos saudáveis e na montagem de uma refeição completa e equilibrada. Ao final da dinâmica, o prato ficou com os idosos, para que eles pudessem visualizar com frequência a refeição completa e continuarem a fazer escolhas saudáveis.

Recursos Humanos: equipe do programa Mesa Brasil e equipe de profissionais do local.

Recursos Materiais: figuras de alimentos saudáveis e não saudáveis, pratos de acrílico, pincel, cola e copos descartáveis. 


\section{DISCUSSÃO DOS RESULTADOS}

Primeiramente coletou-se o resultado do estado nutricional nos prontuários de 13 idosos selecionados para participarem das dinâmicas, classificando-os em baixo peso, eutrófico e sobrepeso/obesidade. Observou-se que 15,38\% $(n=2)$ dos idosos encontram-se em baixo peso, 23,07\% $(n=3)$ em sobrepeso e 61,53\% (n=8) eutrófico. Garcia, Romani e Lira ${ }^{[14]}$ avaliaram o estado nutricional de 308 idosos do nordeste brasileiro e encontraram $14,9 \%$ de idosos desnutridos, 24,4\% sobrepeso e $51,6 \%$ eutrófico, valores aproximados aos encontrados no presente estudo.

Na dinâmica do Bingo dos Hortifrútis foram transmitidas informações sobre a importância nutricional dos vegetais à saúde do idoso e entregue frutas diversas para que eles experimentassem. Todos se divertiram e se interessaram em conhecer seus benefícios. Notou-se que a fruta preferida foi a tangerina (ponkan) e que houve dificuldade para identificar o hortifrúti sorteado com o da cartela, provavelmente, devido às limitações quanto à visão $\mathrm{e}$ memória que acometem a maioria dos idosos.

$\mathrm{Na}$ atividade "Prato Saudável" observou-se que alguns idosos não sabiam quais alimentos formam uma refeição completa e nutritiva e aqueles que devem ser evitados, pois alguns deles colocaram carne em excesso, doces e fast foods (sanduíches, cachorro quente, pizzas, etc.) no prato.

Ao final das dinâmicas, o objetivo foi alcançado, pois eles entenderam a importância de ingerir hortifrútis para a saúde, bem como montar um prato saudável e completo nutricionalmente. Além disso, tiveram a oportunidade de um momento de descontração e, por meio de brincadeiras, puderam exercitar a coordenação motora e a memória.

Em pesquisa realizada por Silva [15], foi observado que os resultados foram semelhantes, pois os idosos tiveram dificuldades de associar o vegetal sorteado com aquele presente na cartela, devido à doença de Alzheimer, que afeta a memória. Com todas estas ações foi possível perceber que estes grupos tiveram algumas dificuldades de memorização e entendimento de temas teóricos, por isso é necessário sempre desenvolver estratégicas lúdicas e pedagógicas, para melhor memorização dos temas estudados e adequação de seus limites e possibilidades de aprendizado para o auto-cuidado.

O Programa Mesa Brasil estabelece uma ponte entre as empresas doadoras de alimentos excedentes e as entidades sociais que precisam deles para suas refeições, com objetivo de combater a fome e o desperdício. Com isso, torna-se possível a elaboração de cardápios saudáveis e nutritivos, completos nutricionalmente, devido a presença de hortifrútis nas preparações, favorecendo grande parte da população em situação de vulnerabilidade social [16].

Por meio da parceria com as instituições, o programa traz qualificação aos funcionários e/ou voluntários, com a realização de ações educativas, oficinas culinárias, cursos e palestras, proporcionando maior conhecimento ao manipulador de alimentos [16].

O Mesa Brasil favorece uma economia significativa às instituições, pois estas conseguem direcionar o valor que seria gasto com estes alimentos, para outros fins, como investir em benfeitorias, aquisição de equipamentos e até no complemento da própria alimentação, promovendo um trabalho de qualidade junto aos institucionalizados ${ }^{[16] .}$

Segundo o Artigo 3o da Lei 11.346/2006, “a segurança alimentar e nutricional é a realização do direito de todos ao acesso regular e permanente de alimentos de qualidade, em quantidade suficiente, sem comprometer o acesso a outras necessidades essenciais, tendo como base práticas promotoras da saúde, que respeitem a diversidade cultural, e que sejam social, econômica e ambientalmente sustentáveis" [17].

Assim, ao reunir esforços de empresas que doam, voluntários que auxiliam e instituições, o Programa Mesa Brasil Sesc demonstra que é possível minimizar os efeitos da fome e da desnutrição por meio de programas sociais e ações educativas.

\section{CONCLUSÃO}

O objetivo do presente trabalho foi alcançado, pois os idosos entenderam sobre os benefícios e a importância dos hortifrútis para a saúde e também como montar uma refeição saudável e completa nutricionalmente. Trabalhou-se também a coordenação motora e o estímulo da memória. Houve dificuldade de assimilar os hortifrútis sorteados com os da cartela, 
porém, ao final da atividade, relembraram dos hortifrútis que consumiam e que conheciam.

Devido ao resultado satisfatório, mesmo na presença das limitações presentes nesse ciclo da vida, notou-se a necessidade e a importância de continuar a atividade educativa nas Instituições de Idosos que este programa social atende. Com isso, conclui-se que a presença do nutricionista é de extrema importância, tendo em vista que este profissional é capacitado para o cálculo da adequação nutricional das refeições que são oferecidas diariamente aos idosos, considerando as alterações fisiológicas e as disfunções degenerativas características desse estágio de vida, de forma a garantir a manutenção da saúde, a prevenção de doenças ou a recuperação destas por meio de uma alimentação saudável. Além disso, o papel do nutricionista é fundamental para o estímulo do consumo de alimentos saudáveis, ricos em nutrientes por meio da realização de ações educativas periódicas.

Sabe-se que um envelhecimento bem sucedido é marcado por uma redução no risco de doenças e pela prevenção ou reversão da perda funcional. O papel deste programa social nas Instituições é de extrema importância, com atenção especial às Instituições que atendem Idosos, levando o bem estar e a qualidade de vida, contribuindo para a segurança alimentar e nutricional e estimulando o desenvolvimento de ações educativas e a promoção de solidariedade.

\section{REFERÊNCIAS}

[1] Sperotto FM, Spinelli RB. Avaliação nutricional em idosos independentes de uma instituição de longa permanência no município de Erechim, RS. Perspectiva. 2010;34:105-116.

[2] Abdala AFV. Nutrição no Envelhecer. São Paulo: Atheneu; 2012.

[3] Segalla R, Spinelli RB. Avaliação nutricional e aplicação de atividades de educação nutricional para idosos institucionalizados em um município do alto Uruguai, RS. Cataventos. 2012;4:97-118.

[4] Segalla R, Spinelli RB. Avaliação e educação nutricional para idosos institucionalizados no município de Erechim, RS. Vivências. 2013;9:77-88.

[5] Ministério da Saúde (BR), Secretaria Nacional de Programas Especiais de Saúde. Portaria no 810, de 22 de setembro de 1989. Aprova normas e os padrões para o funcionamento de casas de repouso, clínicas geriátricas e outras instituições destinadas ao atendimento de idosos, a serem observados em todo o território nacional. Diário Oficial União. 1989.

[6] Toral N, Gubert MB, Schmitz BAS. Perfil da alimentação oferecida em instituições geriátricas do Distrito Federal. Rev. Nutr. 2006;19:29-37.

[7] Campos MTFS, Monteiro JBR, Ornelas APRC. Fatores que afetam o consumo alimentar e a nutrição do idoso. Rev. Nutr. 2000;13:157-165.

[8] Castro CP, Alves CMC, Lopes FF. Fatores sistêmicos associados à doença periodontal em Idosos. RBCEH. 2010;7:289-295.

[9] Carlsson GE. Masticatory efficiency: the effect of age, the loss of teeth and prosthetic rehabilitation. Int Dent J. 1984;34:93-97.

[10] Shuman JM. Nutrição no envelhecimento. In: Mahan LK, Stump SE. Alimentos, nutrição e dietoterapia. São Paulo: Roca; 1998. p.293-312.

[11] Lemos ND, Gazzola JM, Ramos LR. Cuidando do paciente com Alzheimer: o impacto da doença no cuidador. Saúde Social. 2006;15:170-179.

[12] Ballone GJ. Doença de Alzheimer [internet]; 2011 [acesso em 01 mar 2015]. Disponível em: http://www.psiqweb.med.br

[13] Sesc - Serviço Social do Comércio. Mesa São Paulo: ação contra a fome e o desperdício, pela qualidade de vida. São Paulo; 2000.

[14] Garcia ANM, Romani S, Lira PIC. Indicadores antropométricos na avaliação nutricional de idosos: um estudo comparativo. Rev. Nutr. 2007;20:371-378.

[15] Silva MV. Oficina da saúde: Grupos de educação nutricional com idosos portadores de doenças crônicas não transmissíveis na Unidade de Saúde Parque Orlanda. In: IV Mostra Acadêmica UNIMEP [internet]; 2006; Piracicaba. Piracicaba: UNIMEP; 2006 [acesso em 15 jan 2014]. Disponível: http://www.unimep.br/phpg/mostraacademica

[16] Sesc - Serviço Social do Comércio. Mesa Brasil Sesc São Paulo: 10 anos. 2 ed. São Paulo; 2006.

[17] Brasil. Lei no 11.346, de 15 de setembro de 2006. Cria o Sistema Nacional de Segurança Alimentar e Nutricional SISAN com vistas em assegurar o direito humano à alimentação adequada e dá outras providências. Diário Oficial da União. 18 set 2006. 